\title{
Asymptotic Expansions for Product Integration
}

\author{
By Frank de Hoog and Richard Weiss*
}

\begin{abstract}
A generalized Euler-Maclaurin sum formula is established for product integration based on piecewise Lagrangian interpolation. The integrands considered may have algebraic or logarithmic singularities. The results are used to obtain accurate convergence rates of numerical methods for Fredholm and Volterra integral equations with singular kernels.
\end{abstract} form

1. Introduction. A widely used technique for the evaluation of integrals of the

$$
I_{0}(f)=\int_{0}^{1} g(s) f(s) d s,
$$

where $f(t)$ is "smooth" and $g(t)$ is absolutely integrable on $0 \leqq t \leqq 1$, is product integration. This technique consists of replacing $I_{\theta}(f)$ by $I_{\theta}(\tilde{f})$, where $\tilde{f}(t)$ is an approximation to $f(t)$ such that $I_{o}(\tilde{f})$ can be calculated in a simple manner. In this paper, we shall consider a class of such quadrature rules for the case where $g(t)$ may have a finite number of algebraic or logarithmic singularities. These types of singularities are encountered in many applications.

The quadrature rules considered are obtained in the following way: Let

$$
0 \leqq u_{1}<u_{2}<\cdots<u_{n} \leqq 1
$$

be a fixed set of points and define

$$
t_{l}=l h, \quad l=0, \cdots, m ; h=1 / m,
$$

and

$$
t_{l k}=t_{l}+u_{k} h, \quad k=1, \cdots, n ; l=0, \cdots, m-1 .
$$

The approximation $\tilde{f}(t)$ on $t_{l} \leqq t<t_{l+1}, l=0, \cdots, m-1$, is taken to be the $(n-1)$ th degree polynomial interpolating to $f\left(t_{l k}\right), k=1, \cdots, n$.

The main aim of the paper is to establish a generalized Euler-Maclaurin sum formula for the above methods. In Section 2, we describe the quadrature rules in more detail and prove a basic lemma. An Euler-Maclaurin sum formula is established for "smooth" and weakly singular $g(t)$ in Sections 3 and 4, respectively. In Section 5, we apply these results to obtain accurate convergence rates of numerical schemes for Fredholm and Volterra integral equations with singular kernels.

Received March 13, 1972.

AMS (MOS) subject classifications (1970). Primary 65L05.

Key words and phrases. Product integration, asymptotic expansion, Euler-Maclaurin sum formula, integral equation.

* Author's current address: Department of Applied Mathematics, Firestone Laboratories, California Institute of Technology, Pasadena, California 91109.

Copyright (c) 1973, American Mathematical Society 
2. The Product Integration Rule. Define

$$
\omega(t)=\prod_{k=1}^{n}\left(t-u_{k}\right)
$$

and the Lagrangian polynomials

$$
L_{k}(t)=\omega(t) /\left(\omega^{\prime}\left(u_{k}\right)\left(t-u_{k}\right)\right), \quad k=1, \cdots, n .
$$

On $t_{l} \leqq t<t_{l+1}, l=0, \cdots, m-1$, the approximation to $f(t)$ is

$$
\tilde{f}(t)=\sum_{k=1}^{n} L_{k}\left(\frac{t-t_{l}}{h}\right) f\left(t_{l k}\right),
$$

and, hence,

$$
\begin{aligned}
I_{o}(\tilde{f}) & =\sum_{l=0}^{m-1} \int_{t_{l}}^{t_{l+1}} g(s) \tilde{f}(s) d s \\
& =\sum_{l=0}^{m-1} \sum_{k=1}^{n} f\left(t_{l k}\right) \int_{t_{l}}^{t_{l+1}} g(s) L_{k}\left(\frac{s-t_{l}}{h}\right) d s \\
& =\sum_{l=0}^{m-1} \sum_{k=1}^{n} h f\left(t_{l k}\right) \int_{0}^{1} g\left(t_{l}+s h\right) L_{k}(s) d s .
\end{aligned}
$$

This is the $n m$ point quadrature rule with which this paper is concerned. The weights are calculated by evaluating the integrals analytically. The error functional for this rule is

$$
E_{o}(f)=I_{o}(\tilde{f})-I_{o}(f)=I_{0}(\tilde{f}-f) .
$$

In the following lemma, an expression for the error functional is obtained.

LEMMA 2.1. If $f(t) \in C^{p+1}[0, T], p \geqq n$, then

$$
E_{g}(f)=\sum_{r=0}^{p-n} h^{n+r} \int_{0}^{1} \omega_{r}(s) h \sum_{l=0}^{m-1} g\left(t_{l}+s h\right) f^{(n+r)}\left(t_{l}+s h\right) d s+O\left(h^{p+1}\right)
$$

where

$$
\omega_{r}(t)=\omega(t) p_{r}(t)
$$

and $p_{r}(t)$ is a polynomial of degree $r$.

Proof. It is clear that

$$
E_{0}(f)=h \sum_{l=0}^{m-1} \int_{0}^{1} g\left(t_{l}+s h\right)\left\{\tilde{f}\left(t_{l}+s h\right)-f\left(t_{l}+s h\right)\right\} d s .
$$

For $0 \leqq s \leqq 1$, it follows from (1.1) and Taylor's theorem that

$$
\begin{aligned}
f\left(t_{l k}\right)= & f\left(t_{l}+s h-\left(s-u_{k}\right) h\right) \\
& =\sum_{r=0}^{p} h^{r} \frac{(-1)^{r}\left(s-u_{k}\right)^{r}}{r !} f^{(r)}\left(t_{l}+s h\right)+O\left(h^{p+1}\right), \\
& \quad k=1, \cdots, n ; l=0, \cdots, m-1 .
\end{aligned}
$$

Hence, 


$$
\begin{aligned}
& \tilde{f}\left(t_{l}+s h\right)-f\left(t_{l}+s h\right)=\sum_{k=1}^{n}\left\{f\left(t_{l k}\right)-f\left(t_{l}+s h\right)\right\} L_{k}(s) \\
&=\sum_{r=1}^{p} h^{r} \frac{(-1)^{r}}{r !} f^{(r)}\left(t_{l}+s h\right) \sum_{k=1}^{n}\left(s-u_{k}\right)^{r} L_{k}(s)+O\left(h^{p+1}\right), \\
& \quad l=0, \cdots, m-1 .
\end{aligned}
$$

Since

$$
\sum_{k=1}^{n}\left(s-u_{k}\right)^{r} L_{k}(s)=\omega(s) \sum_{k=1}^{n}\left(s-u_{k}\right)^{r-1} / \omega^{\prime}\left(u_{k}\right)
$$

and

$$
\sum_{k=1}^{n} u_{k}^{a} / \omega^{\prime}\left(u_{k}\right)=0, \quad q=0, \cdots, n-2
$$

it follows that

$$
\sum_{k=1}^{n}\left(s-u_{k}\right)^{r} L_{k}(s)=0, \quad r=0, \cdots, n-1
$$

For $r \geqq n-1$,

$$
\begin{aligned}
\sum_{k=1}^{n} \frac{\left(s-u_{k}\right)^{r}}{\omega^{\prime}\left(u_{k}\right)} & =\sum_{q=0}^{r}\left(\begin{array}{l}
r \\
q
\end{array}\right)(-1)^{q} s^{r-a} \sum_{k=1}^{n} \frac{u_{k}^{a}}{\omega^{\prime}\left(u_{k}\right)} \\
& =\sum_{q=n-1}^{r} \sum_{k=1}^{n}\left(\begin{array}{l}
r \\
q
\end{array}\right)(-1)^{a} \frac{u_{k}^{q}}{\omega^{\prime}\left(u_{k}\right)} s^{r-q} .
\end{aligned}
$$

Substitution of (2.7), (2.8) and (2.9) into (2.6) yields

$$
\begin{array}{r}
\tilde{f}\left(t_{l}+s h\right)-f\left(t_{l}+s h\right)=\sum_{r=0}^{p-n} h^{n+r} f^{(n+r)}\left(t_{l}+s h\right) \omega(s) p_{r}(s)+O\left(h^{p+1}\right), \\
l=0, \cdots, m-1,
\end{array}
$$

where

$$
p_{r}(s)=\frac{(-1)^{r}}{(n+r) !} \sum_{a=0}^{r} \sum_{k=1}^{n}\left(\begin{array}{l}
n+r-1 \\
n+q-1
\end{array}\right)(-1)^{q-1} \frac{u_{k}^{n+q-1}}{\omega^{\prime}\left(u_{k}\right)} s^{r-a} .
$$

The result follows on substituting (2.10) into (2.5).

Remark. Clearly, $\omega_{r}(t), r=0, \cdots, p-n$, also depend on $u_{k}, k=1, \cdots, n$. In addition, it should be noted that Lemma 2.1 is valid for any absolutely integrable $g(t)$.

For fixed $s, 0 \leqq s \leqq 1$, the sum

$$
h \sum_{l=0}^{m-1} g\left(t_{l}+s h\right) f^{(n+r)}\left(t_{l}+s h\right)
$$

is a generalized Euler approximation to $\int_{0}^{1} g(s) f^{(n+r)}(s) d s$.

Summation formulae for (2.11) have been investigated by Lyness and Ninham [4] and the application of their results to (2.3) is the basis of Section 4 . 
3. Smooth $g(t)$. Let $f(t) \in C^{p+1}[0, T], p \geqq n$ and $g(t) \in C^{p-n+1}[0, T]$. Applying the Euler-Maclaurin sum formula to $g(t) f^{(n+r)}(t)$, we find

$$
\begin{aligned}
& h \sum_{l=0}^{m-1} g\left(t_{l}+x h\right) f^{(n+r)}\left(t_{l}+x h\right)=\int_{0}^{1} g(s) f^{(n+r)}(s) d s \\
& \quad+\sum_{q=0}^{p-n-r-1} h^{q+1} \frac{B_{q+1}(x)}{(q+1) !}\left[\left.\frac{d^{q}}{d t^{q}}\left(g(t) f^{(n+r)}(t)\right)\right|_{t=1}-\left.\frac{d^{Q}}{d t^{q}}\left(g(t) f^{(n+r)}(t)\right)\right|_{t=0}\right] \\
& \quad+O\left(h^{p-n-r+1}\right), \quad r=0, \cdots, p-n,
\end{aligned}
$$

where $B_{q}(x), q=1,2, \cdots$, are the Bernoulli polynomials. Substituting (3.1) into (2.3) and collecting powers of $h$, we obtain

$$
\begin{aligned}
& E_{0}(f)=h^{n} \int_{0}^{1} \omega_{0}(s) d s \int_{0}^{1} g(s) f^{(n)}(s) d s \\
& +\sum_{r=0}^{p-n-1} h^{n+r+1}\left\{\int_{0}^{1} \omega_{r+1}(s) d s \int_{0}^{1} g(s) f^{(n+r+1)}(s) d s\right. \\
& \quad+\sum_{l=0}^{r} \frac{1}{(1+r-l) !}\left[\left.\frac{d^{r-l}}{d t^{r-l}}\left(g(t) f^{(n+l)}(t)\right)\right|_{t=1}\right. \\
& \left.\left.\quad-\left.\frac{d^{r-l}}{d t^{r-l}}\left(g(t) f^{(n+l)}(t)\right)\right|_{t=0}\right] \int_{0}^{1} \omega_{l}(s) B_{1+r-l}(s) d s\right\} \\
& +O\left(h^{p+1}\right) .
\end{aligned}
$$

The above equation is a generalized Euler-Maclaurin expansion for the error functional.

If $u_{k}, k=1, \cdots, n$, are chosen such that

$$
\int_{0}^{1} s^{r} \omega(s) d s=0, \quad r=0,1, \cdots, q<n,
$$

it is clear from (2.4) that

$$
\int_{0}^{1} s^{l} \omega_{r}(s) d s=0, \quad r=0,1, \cdots, q ; l=0, \cdots, q-r,
$$

and, hence, the first $q+1$ terms in (3.2) vanish. This may be expected since, for $g(t)=1$, (3.2) reduces to the Euler-Maclaurin sum formula for the corresponding composite interpolatory quadrature rule (see, for instance, Baker and Hodgson [2]).

In the case that $g(t)=1$ and a symmetric rule is used, the coefficients of the odd powers of $h$ are zero, and so the expansion is in integer powers of $h^{2}$. It should be noted that, for general $g(t)$, this does not happen, as, in general, the rule is not symmetric.

4. Singular $g(t)$. In this section, we shall consider the case where $g(t)$ has a finite number of algebraic or logarithmic singularities.

Firstly, we shall establish an Euler-Maclaurin sum formula when

$$
g(t)=t^{\beta}(1-t)^{\omega}\left|t-v_{k}\right|^{\gamma} \operatorname{sgn}\left(t-v_{i}\right)\left|t-v_{i}\right|^{\delta}, \quad \beta, \omega, \gamma, \delta>-1 .
$$


As in Section 3, expansions for sums of the form

$$
h \sum_{l=0}^{m-1} g\left(t_{l}+x h\right) z\left(t_{l}+x h\right),
$$

where $z(t)$ is a smooth function, are required. Such expansions have been derived by Lyness and Ninham [4] who use Lighthill's procedure to obtain asymptotic expansions for the integral terms in Poisson's sum formula [4, Eq. (3.13)],

$$
\begin{aligned}
h \sum_{l=0}^{m-1} g\left(t_{l}+\right. & x h) z\left(t_{l}+x h\right)-\int_{0}^{1} g(s) z(s) d s \\
& =\sum_{q=-\infty}^{+\infty}(-1)^{a} \exp (-\pi i(2 x-1) q) \int_{0}^{1} g(s) z(s) \exp (2 \pi i q m s) d s \\
& =\sum_{q=-\infty}^{+\infty} \exp (-2 \pi i q x) \int_{0}^{1} g(s) z(s) \exp (2 \pi i q s / h) d s .
\end{aligned}
$$

Applying the results of Lyness and Ninham [4, Eq. (8.1)] to $g(t) f^{(n+r)}(t)$, we find that

$$
h \sum_{l=0}^{m-1} g\left(t_{l}+x h\right) f^{(n+r)}\left(t_{l}+x h\right)=\int_{0}^{1} g(s) f^{(n+r)}(s) d s+\sum_{q=0}^{p-n-r} \frac{h^{a+1}}{q !}
$$

$$
\begin{aligned}
& \cdot\left\{h^{\beta} \tilde{\zeta}(-\beta-q, x) \psi_{0 r}^{(\alpha)}(0)\right. \\
& \quad+h^{\omega}(-1)^{q} \tilde{\zeta}(-\omega-q, 1-x) \psi_{1 r}^{(a)}(1) \\
& \quad+h^{\gamma}\left(\xi\left(-\gamma-q, x-m v_{k}\right)+(-1)^{q} \tilde{\zeta}\left(-\gamma-q, m v_{k}-x\right)\right) \psi_{2 r}^{(a)}\left(v_{k}\right) \\
& \left.\quad+h^{\delta}\left(\xi\left(-\delta-q, x-m v_{i}\right)-(-1)^{a} \tilde{\zeta}\left(-\delta-q, m v_{i}-x\right)\right) \psi_{3 r}^{(a)}\left(v_{i}\right)\right\} \\
& +O\left(h^{p-n-r+1}\right), \quad r=0, \cdots, p-n
\end{aligned}
$$

where

$$
\begin{aligned}
& \psi_{0 r}(t)=f^{(n+r)}(t)(1-t)^{\omega}\left|t-v_{k}\right|^{\gamma} \operatorname{sgn}\left(t-v_{i}\right)\left|t-v_{i}\right|^{\delta}, \\
& \psi_{1 r}(t)=f^{(n+r)}(t) t^{\beta}\left|t-v_{k}\right|^{\gamma} \operatorname{sgn}\left(t-v_{i}\right)\left|t-v_{i}\right|^{\delta}, \\
& \psi_{2 r}(t)=f^{(n+r)}(t) t^{\beta}(1-t)^{\omega} \operatorname{sgn}\left(t-v_{i}\right)\left|t-v_{i}\right|^{\delta}, \\
& \psi_{3 r}(t)=f^{(n+r)}(t) t^{\beta}(1-t)^{\omega}\left|t-v_{k}\right|^{\gamma},
\end{aligned}
$$

and $\bar{\zeta}(\alpha, x)$ is the periodic generalized zeta function. The periodic generalized zeta function is defined by

$$
\xi(\alpha, x)=\zeta(\alpha, \tilde{x}), \quad x-\tilde{x}=\text { integer, } \quad 0<\tilde{x} \leqq 1,
$$

where $\zeta(\alpha, x)$ is the generalized Riemann zeta function (see, for instance, Whittaker and Watson [6]).

Substitution of (4.3) into (2.3) yields 


$$
\begin{aligned}
& E_{o}(f)=\sum_{r=0}^{p-n} h^{n+r} \int_{0}^{1} \omega_{r}(s) d s \int_{0}^{1} g(s) f^{(n+r)}(s) d s \\
& +\sum_{r=0}^{p-n} h^{n+r+\beta+1} \sum_{l=0}^{r} \frac{\psi_{0 l}^{(r-l)}(0)}{(r-l) !} \int_{0}^{1} \omega_{l}(s) \tilde{\zeta}(-\beta-r+l, s) d s \\
& +\sum_{r=0}^{p-n} h^{n+r+\omega+1} \sum_{l=0}^{r} \frac{(-1)^{r-l} \psi_{1 l}^{(r-l)}(1)}{(r-l) !} \int_{0}^{1} \omega_{l}(s) \tilde{\zeta}(-\omega-r+l, 1-s) d s \\
& +\sum_{r=0}^{p-n} h^{n+r+\gamma+1} \sum_{l=0}^{r} \frac{\psi_{2 l}^{(r-l)}\left(v_{k}\right)}{(r-l) !} \int_{0}^{1} \omega_{l}(s)\left[\tilde{\zeta}\left(-\gamma-r+l, s-m v_{k}\right)\right. \\
& \quad+\sum_{r=0}^{p-n} h^{n+r+\delta+1} \sum_{l=0}^{r} \frac{\psi_{3 l}^{(r-l)}\left(v_{i}\right)}{(r-l) !} \int_{0}^{1} \omega_{l}(s)\left[\tilde{\zeta}\left(-\delta-r+l, s-m v_{i}\right)\right. \\
& \\
& +
\end{aligned}
$$

This is the desired Euler-Maclaurin expansion for $g(t)$ given by (4.1). For the important case of endpoint singularities (i.e., $\left.g(t)=t^{\beta}(1-t)^{\omega}\right)$, terms of the form $\int_{0}^{1} \omega_{l}(s)$. $\tilde{\zeta}(\dot{\alpha}, s) d s$ and $\int_{0}^{1} \omega_{l}(s) \tilde{\zeta}(\alpha, 1-s) d s$ can be reduced to sums of ordinary zeta functions by the relations

$$
\int_{0}^{1} \zeta(\alpha, s) d s=0, \quad \alpha<1
$$

and

$$
\begin{aligned}
& \int_{0}^{1} s^{r} \zeta(\alpha, s) d s=\frac{1}{1-\alpha}\left(\zeta(\alpha-1)-r \int_{0}^{1} s^{r-1} \zeta(\alpha-1, s) d s\right) \\
& r=1,2, \cdots ; \alpha<1 .
\end{aligned}
$$

If $u_{k}, k=1, \cdots, n$, are chosen such that

$$
\int_{0}^{1} \omega(s) d s=0
$$

the first term in (4.4) is deleted. However, in general, (3.3) does not lead to higher order convergence. From (4.4) it is clear that the conditions required depend on $g(t)$.

To illustrate this, we take $g(t)=t^{-1 / 2}$ and determine the conditions necessary for optimal convergence in the cases $n=2$ and $n=3$.

If $n=2$, we require (4.5) and

$$
\int_{0}^{1} \omega(s) \tilde{\zeta}\left(\frac{1}{2}, s\right) d s=0
$$

Numerical calculation yields

$$
u_{1}=.1182506123, \quad u_{2}=.7182932992 .
$$

For $n=3$, we require (4.5), (4.6) and

$$
\int_{0}^{1} s \omega(s) d s=0 .
$$


Numerical calculation yields

(4.8) $\quad u_{1}=.04456270208$,

$$
u_{2}=.3909749362, \quad u_{3}=.8537066313 .
$$

The quadrature formulae with the points given by (4.7) and (4.8) have been applied to

$$
I_{0}(f)=\int_{0}^{1} \frac{(2-x)^{1 / 2}}{x^{1 / 2}} d x=1+\pi / 2
$$

Numerical results for various stepsizes are tabulated in Table 1. The order of convergence can be seen to be three and four and a half, respectively.

Remark. All computations were done in double-precision arithmetic on the IBM 360/50 computer at the Australian National University.

The extension of (4.4) to a $g(t)$ which includes terms of the form $\ln t, \ln (1-t)$, $\ln \left|t-v_{k}\right|$ and sgn $\left(t-v_{i}\right) \ln \left|t-v_{i}\right|$ can be made by differentiation with respect to $\beta, \omega, \gamma$ and $\delta$, respectively. To illustrate this, we consider the case when

$$
g(t)=\ln \left|t-v_{k}\right|=\left.\frac{\partial}{\partial \gamma}\left(\left|t-v_{k}\right|^{\gamma}\right)\right|_{\gamma=0}, \quad 0<v_{k}<1 .
$$

Then

$$
\begin{aligned}
& E_{0}(f)=h^{n} \int_{0}^{1} \omega_{0}(s) d s \int_{0}^{1} g(s) f^{(n)}(s) d s \\
& +\sum_{r=0}^{p-n-1} h^{n+r-1}\left\{\int_{0}^{1} \omega_{r+1}(s) d s \int_{0}^{1} g(s) f^{(n+r+1)}(s) d s\right. \\
& +\sum_{l=0}^{r} \frac{1}{(r-l) !}\left[\left.\frac{d^{r-1}}{d t^{r-l}}\left(g(t) f^{(n+l)}(t)\right)\right|_{t=0} \int_{0}^{1} \omega_{l}(s) \tilde{\xi}(-r+l, s) d s\right. \\
& +\left.(-1)^{r-l} \frac{d^{r-l}}{d t^{r-l}}\left(g(t) f^{(n+l)}(t)\right)\right|_{t=1} \\
& \left.\left.\cdot \int_{0}^{1} \omega_{l}(s) \tilde{\xi}(-r+l, 1-s) s d\right]\right\} \\
& +\sum_{r=0}^{p-n} f^{(n+r)}\left(v_{k}\right) h^{n+r+1}\left\{\operatorname { l n } h \sum _ { l = 0 } ^ { r } \frac { 1 } { ( r - l ) ! } \int _ { 0 } ^ { 1 } \omega _ { l } ( s ) \left(\tilde{\zeta}\left(-r+l, s-m v_{k}\right)\right.\right. \\
& \left.+(-1)^{r-1} \tilde{\zeta}\left(-r+l, m v_{k}-s\right)\right) d s \\
& +\sum_{l=0}^{r} \frac{1}{(r-l) !} \int_{0}^{1} \omega_{l}(s)\left(\tilde{\zeta}^{\prime}\left(-r+l, s-m v_{k}\right)\right. \\
& \left.\left.+(-1)^{r-l} \tilde{\zeta}^{\prime}\left(-r+l, m v_{k}-s\right)\right) d s\right\} \\
& +O\left(h^{p+1}\right)
\end{aligned}
$$

where

$$
\tilde{\zeta}^{\prime}(\alpha, s)=-\partial \tilde{\xi}(\alpha, s) / \partial \alpha
$$


This expansion can be simplified slightly by substitution of the relations

$$
\zeta(-q, s)=-B_{q+1}(s) /(q+1), \quad q=0,1,2, \cdots .
$$

Again, if (4.5) holds, the first term in (4.9) is deleted.

TABLE 1

\begin{tabular}{lcc}
\hline Stepsize & $n=2$ & $n=3$ \\
$h$ & $E_{o}(f)$ & $E_{o}(f)$ \\
\hline 0.2 & 6.008 E-6 & 3.025 E-9 \\
0.1 & 7.004 E-7 & 1.505 E-10 \\
0.05 & 8.287 E-8 & 6.956 E-12 \\
0.025 & 9.933 E-9 & 3.013 E-13 \\
\hline
\end{tabular}

5. The Application to Integral Equations. Atkinson [1] considers the numerical solution of linear Fredholm integral equations of the second kind with singular kernels

$$
y(t)=G(t)+\lambda \int_{0}^{1} K(t, s) y(s) d s, \quad 0 \leqq t \leqq 1,
$$

where

$$
K(t, s)=\sum_{k=1}^{r} P_{k}(t, s) Q_{k}(t, s), \quad r \geqq 1,
$$

and $P_{k}(t, s), Q_{k}(t, s), k=1, \cdots, r$, satisfy

(i) $Q_{k}(t, s)$ is continuous on $0 \leqq s, t \leqq 1$;

(ii) $\int_{0}^{1}\left|P_{k}(t, s)\right| d s$ is bounded;

(iii) $\lim _{\left|t_{1}-t_{2}\right| \rightarrow 0} \int_{0}^{1}\left|P_{k}\left(t_{1}, s\right)-P_{k}\left(t_{2}, s\right)\right| d s=0$ uniformly in $t_{1}$ and $t_{2}$.

Important cases of $P_{k}(t, s)$ are

$$
|t-s|^{\gamma},|v-s|^{\gamma}, \quad 0>\gamma>-1, \quad \ln |t-s|, \ln |v-s|, \quad 0 \leqq v \leqq 1 .
$$

For illustrative purposes, it is sufficient to consider the case

$$
K(t, s)=P(t, s) Q(t, s) .
$$

The application of product integration to the integral term in (5.1) yields the numerical scheme

$$
\begin{aligned}
Y_{i j}=G\left(t_{i j}\right)+\lambda \sum_{l=0}^{m-1} \sum_{k=1}^{n} W_{l k}\left(t_{i j}\right) Q\left(t_{i j}, t_{l k}\right) Y_{l k}, & \\
& j=1, \cdots, n ; i=0, \cdots, m-1,
\end{aligned}
$$

where

$$
W_{l k}(t)=\int_{t_{l}}^{t_{l+1}} P(t, s) L_{k}\left(\frac{s-t_{l}}{h}\right) d s
$$

and $Y_{i j}$ denotes the numerical approximation to $y\left(t_{i j}\right)$. Atkinson has shown that 
if $\lambda$ is not an eigenvalue of (5.1), then (5.4) has a unique solution for sufficiently small $h$ and

$$
\max _{j=1, \cdots, n ; i=0, \cdots, m-1}\left|y\left(t_{i j}\right)-Y_{i j}\right|=O(E),
$$

where

$$
\begin{aligned}
E=\max _{i=1, \ldots, n ; i=0, \cdots, m-1} \mid \sum_{l=0}^{m-1} \sum_{k=1}^{n} W_{l k}\left(t_{i j}\right) Q\left(t_{i i}, t_{l k}\right) y\left(t_{l k}\right) & \\
& -\int_{0}^{1} K\left(t_{i i}, s\right) y(s) d s \mid .
\end{aligned}
$$

We shall now indicate how the results of Section 4 can be extended to obtain accurate estimates for (5.5). It will be assumed that $Q(t, s) y(s)$ is $p+1$ times continuously differentiable with respect to $s$.

The direct application of the results in Section 4 yields the following estimates for $E$ :

$$
E=O\left(h^{n} \int_{0}^{1} \omega(s) d s\right)+O\left(h^{n+1+\gamma}\right) \text { for } P(t, s)=|v-s|^{\gamma}
$$

and

$$
E=O\left(h^{n} \int_{0}^{1} \omega(s) d s\right)+O\left(h^{n+1} \ln h\right) \text { for } P(t, s)=\ln |v-s| .
$$

However, for the case when $P(t, s)=|t-s|^{\gamma}$ or $\ln |t-s|,(4.4)$ and (4.9) are no longer valid since $v_{k}$ takes the values $t_{i i}, j=1, \cdots, n ; i=0, \cdots, m-1$, and thus depends on $h$. The extension of the results of Section 4 to these cases is obtained in the following way. First, the integral terms in (4.2) are rewritten as

$\int_{0}^{1} g(s) z(s) \exp \left(\frac{2 \pi i q s}{h}\right) d s=t_{i j} \int_{0}^{1} g\left(t_{i j} s\right) z\left(t_{i} s\right) \exp \left(\frac{2 \pi i q t_{i j} s}{h}\right) d s$

$$
\begin{array}{r}
+\left(1-t_{i i}\right) \exp \left(\frac{2 \pi i q t_{i j}}{h}\right) \int_{0}^{1} g\left(\left(1-t_{i j}\right) s+t_{i j}\right) z\left(\left(1-t_{i j}\right) s+t_{i i}\right) \\
\cdot \exp \left(2 \pi i q\left(1-t_{i i}\right) s / h\right) d s, \\
j=1, \cdots, n ; \quad i=0, \cdots, m-1 .
\end{array}
$$

For

$$
g(t)=\left|t_{i i}-t\right|^{\gamma}, \quad 0<t_{i j}<1,
$$

Eq. (5.6) becomes

$$
\begin{aligned}
& \int_{0}^{1} g(s) z(s) \exp \left(\frac{2 \pi i q s}{h}\right)=t_{i j}^{1+\gamma} \int_{0}^{1}(1-s)^{\gamma} z\left(t_{i j} s\right) \exp \left(\frac{2 \pi i q s}{\hat{h}}\right) d s \\
& +\left(1-t_{i j}\right)^{1+\gamma} \exp \left(\frac{2 \pi i q}{\hat{h}}\right) \int_{0}^{1} s^{\gamma} z\left(\left(1-t_{i j}\right) s+t_{i i}\right) \exp \left(\frac{2 \pi i q s}{\hat{h}}\right) d s, \\
& \quad 0<t_{i i}<1,
\end{aligned}
$$


where

$$
h=h / t_{i j} ; \quad \hat{h}=h /\left(1-t_{i j}\right) .
$$

The singularities of the integrands on the right-hand side of (5.8) are now endpoint singularities independent of $t_{i j}$ and so asymptotic expansions in $\hat{h}$ and $\hat{h}$, respectively, for the corresponding integrals can be calculated in a similar way to [4] by Lighthill's procedure.

Define

$$
G_{1}\left(t_{i i}, \tilde{\tau}\right)=\int_{0}^{1}(1-s)^{\gamma} z\left(t_{i} s\right) \exp (2 \pi i \tilde{\tau} s) d s
$$

and

$$
G_{2}\left(t_{i i}, \hat{\tau}\right)=\int_{0}^{1} s^{\gamma} z\left(\left(1-t_{i j}\right) s+t_{i j}\right) \exp (2 \pi i \hat{i} s) d s
$$

where

$$
\tilde{\tau}=q / h ; \quad \hat{\tau}=q / \hat{h} ; \quad q=0,1,2, \cdots .
$$

Clearly, $G_{1}\left(t_{i}, \tau\right)$ and $G_{2}\left(t_{i j}, \tau\right)$ are the Fourier transforms of the generalized functions

$$
\phi_{1}\left(t_{i i}, s\right)=(1-s)^{\gamma} z\left(t_{i} s\right) H(s) H(1-s)
$$

and

$$
\phi_{2}\left(t_{i i}, s\right)=s^{\gamma} z\left(\left(1-t_{i j}\right) s+t_{i j}\right) H(s) H(1-s)
$$

where $H$ is the Heaviside step function defined by

$$
H(s)= \begin{cases}1, & s>0, \\ \frac{1}{2}, & s=0, \\ 0, & s<0 .\end{cases}
$$

For $k \geqq 0$, let

$$
\begin{aligned}
& \psi_{1}\left(t_{i i}, s\right)=(1-s)^{\gamma} z\left(t_{i j} s\right) \\
& \psi_{2}\left(t_{i i}, s\right)=s^{\gamma} z\left(\left(1-t_{i j}\right) s+t_{i j}\right) \\
& R_{1}\left(t_{i i}, s\right)=\sum_{a=0}^{k} \frac{1}{q !} \frac{\partial^{q} \psi_{1}}{\partial s^{q}}\left(t_{i i}, 0\right) s^{q} H(s) \\
& R_{2}\left(t_{i i}, s\right)=\sum_{q=0}^{k} \frac{\left(-t_{i j}\right)^{q}}{q !} z^{(q)}\left(t_{i j}\right)(1-s)^{q+\gamma} H(1-s) \\
& R_{3}\left(t_{i i}, s\right)=\sum_{q=0}^{k} \frac{\left(1-t_{i j}\right)^{q}}{q !} z^{(q)}\left(t_{i i}\right) s^{q+\gamma} H(s)
\end{aligned}
$$

and

$$
R_{4}\left(t_{i i}, s\right)=\sum_{q=0}^{k} \frac{(-1)^{a}}{q !} \frac{\partial^{a} \psi_{2}}{\partial s^{a}}\left(t_{i i}, 1\right)(1-s)^{q} H(1-s) .
$$


Then it follows from Lighthill's theorem that

$$
G_{1}\left(t_{i j}, \tilde{\tau}\right)=\int_{-\infty}^{+\infty}\left\{R_{1}\left(t_{i i}, s\right)+R_{2}\left(t_{i i}, s\right)\right\} \exp (2 \pi i \tilde{\tau} s) d s+O\left(|\tilde{\tau}|^{-k-1}\right)
$$

and

$$
G_{2}\left(t_{i i}, \hat{\tau}\right)=\int_{-\infty}^{+\infty}\left\{R_{3}\left(t_{i i}, s\right)+R_{4}\left(t_{i i}, s\right)\right\} \exp (2 \pi i \hat{\tau} s) d s+O\left(|\hat{\tau}|^{-k-1}\right)
$$

The generalized Fourier transforms in (5.9) and (5.10) can be evaluated by the standard integrals given in [4, Eq. (6.14)]. Substituting the resulting asymptotic expansions for $G_{1}\left(t_{i j}, q / \tilde{h}\right)$ and $G_{2}\left(t_{i}, q / \hat{h}\right), q=0,1, \cdots$, into (4.2), we obtain in the same way as [4]

$$
\begin{aligned}
& h \sum_{l=0}^{m-1}\left|t_{l}+x h-t_{i j}\right|^{\gamma} z\left(t_{l}+x h\right)=\int_{0}^{1}\left|s-t_{i i}\right|^{\gamma} z(s) d s \\
& +\sum_{a=0}^{k} \frac{h^{a+1}}{q !}\left\{\left.\xi(-q, x) \frac{d^{q}}{d t^{a}}\left(\left|t-t_{i j}\right|^{\gamma} z(t)\right)\right|_{t=0}\right. \\
& \left.+\left.(-1)^{a} \tilde{\zeta}(-q, 1-x) \frac{d^{q}}{d t^{a}}\left(\left|t-t_{i j}\right|^{\gamma} z(t)\right)\right|_{t=1}\right\} \\
& +\sum_{a=0}^{k} \frac{h^{a+1+\gamma}}{q !}\left\{\xi\left(-\gamma-q, x-u_{j}\right)+(-1)^{a} \xi\left(-\gamma-q, 1+u_{i}-x\right)\right\} z^{(a)}\left(t_{i j}\right) \\
& +O\left(t_{i j}^{1+\gamma} h^{k+1}\right)+O\left(\left(1-t_{i j}\right)^{1+\gamma} \hat{h}^{k+1}\right), \quad 0<t_{i j}<1, k \geqq 0 .
\end{aligned}
$$

Hence, it is easy to verify that for $g(t)$ defined by (5.7), Eq. (4.4) remains valid if the order term is replaced by

$$
O\left(h^{p+1} / t_{i i}^{p-n-\gamma}\right)+O\left(h^{p+1} /\left(1-t_{i i}\right)^{p-n-\gamma}\right) .
$$

In a similar way, it can be shown that for $g(t)=\ln \left|t-t_{i j}\right|$ the order terms in (4.9) have to be replaced by

$$
O\left(\ln \left(t_{i j}\right) h^{p+1} / t_{i j}^{p-n}\right)+O\left(\ln \left(1-t_{i j}\right) h^{p+1} /\left(1-t_{i j}\right)^{p-n}\right) .
$$

We thus obtain the estimates

$$
E=O\left(h^{n} \int_{0}^{1} \omega(s) d s\right)+O\left(h^{n+1+\gamma}\right) \text { for } P(t, s)=|t-s|^{\gamma},
$$

and

$$
E=O\left(h^{n} \int_{0}^{1} \omega(s) d s\right)+O\left(h^{n+1} \ln h\right) \quad \text { for } P(t, s)=\ln |t-s|
$$

As an example, consider the equation

$$
y(t)=1+\int_{0}^{\pi} \sum_{k=1}^{4} P_{k}(t, s) Q_{k}(t, s) y(s) d s, \quad 0 \leqq t \leqq \pi,
$$


where

$$
\begin{aligned}
& Q_{1}(t, s)=\left\{\frac{\sin ((t-s) / 2)}{((t-s) / 2)}\right\}+\ln \left\{\frac{\sin ((t+s) / 2)}{(t+s)(2 \pi-t-s)}\right\}, \\
& P_{2}(t, s)=\ln |t-s|, \quad P_{3}(t, s)=\ln (2 \pi-t-s), \\
& P_{4}(t, s)=\ln (t+s), \quad P_{1}=Q_{2}=Q_{3}=Q_{4}=1,
\end{aligned}
$$

which has the solution

$$
y(t)=1 /(1+\pi \ln 2) .
$$

Atkinson [1] has applied the product Simpson rule $\left(u_{1}=0, u_{2}=\frac{1}{2}, u_{3}=1, \int_{0}^{1} \omega(s) d s\right.$ $=0)$ to this equation. Although the rate of convergence was observed to be approximately $O\left(h^{4}\right)$, only $O\left(h^{3}\right)$ convergence was established. The above estimates yield $O\left(h^{4} \ln h\right)$ convergence.

The above can also be extended to Volterra integral equations of the second kind with singular kernels. Linz [3] applies a product Simpson and a product block by block method based on the points $u_{1}=0, u_{2}=\frac{1}{2}, u_{3}=1$ to the equation

$$
y(t)=G(t)+\int_{0}^{t} \frac{K(t, s, y(s))}{(t-s)^{1 / 2}} d s, \quad t \geqq 0,
$$

and estimates order three convergence. The correct order for both methods is three and a half.

Remark. The extension of (4.4) (and hence (5.1)) to the general case with singularities of the form (4.1) where $v_{k}, v_{i}$ may depend linearly on $h$ can be made by a splitting similar to the above and a similar analysis to that given in Ninham and Lyness [5]. The details of such an analysis however are beyond the scope of this paper.

Acknowledgements. The authors gratefully acknowledge the helpful comments given by Professor B. W. Ninham during the preparation of this paper. In addition, they wish to thank Dr. M. R. Osborne and the referee for their valuable criticism of the first version of the paper.

\section{Computer Centre}

The Australian National University

Canberra A.C.T. 2600, Australia

1. K. E. Atkinson, "The numerical solution of Fredholm integral equations of the second kind," SIAM J. Numer. Anal., v. 4, 1967, pp. 337-348. MR 36 \#7358.

2. C. T. H. BaKer \& G. S. Hodgson, "Asymptotic expansions for integration formulas in one or more dimensions," SIAM J. Numer. Anal., v. 8, 1971, pp. 473-480. MR 44 \#2339.

3. P. LINZ, "Numerical methods for Volterra integral equations with singular kernels," SIAM J. Numer. Anal., v. 6, 1969 , pp. 365-374. MR 41 \#4850.

4. J. N. LyNESS \& B. W. NinhaM, "Numerical quadrature and asymptotic expansions," Math. Comp., v. 21, 1967, pp. 162-178. MR 37 \#1081.

5. B. W. NinHAM \& J. N. LYNESS, "Further asymptotic expansions for the error functional," Math. Comp., v. 23, 1969, pp. 71-83. MR $39 \# 3682$.

6. E. T. Whittaker \& G. N. Watson, A Course of Modern Analysis, Cambridge Univ. Press, New York, 1958. 